\title{
1 Optimizing the live attenuated influenza A vaccine backbone for high-risk patient groups
}

2 João P.P. Bonifacio ${ }^{1}$, Nathalia Williams ${ }^{1}$, Laure Garnier ${ }^{2}$, Stephanie Hugues ${ }^{2,3}$, Mirco

3 Schmolke ${ }^{1,3}$, Beryl Mazel-Sanchez ${ }^{1 *}$

4

$5{ }^{1}$ Department of Microbiology and Molecular Medicine, University of Geneva, Geneva, 1211,

6 Switzerland

7 2Department of Microbiology and Molecular Medicine, University of Geneva, Geneva, 1211,

8 Switzerland

$9{ }^{3}$ Geneva Center for inflammation research (GCIR) University of Geneva, Geneva, 1211,

10 Switzerland

11 *correspondence: Beryl.Mazel-Sanchez@unige.ch

\section{Abstract (145/150)}

14 The live attenuated influenza vaccine (LAIV) is approved for intranasal spray application in 249 year-old patients with safety concerns limiting its use in younger children and immunocompromised patients, mainly from the higher incidence of adverse events and the

17 possibility of uncontrolled replication and reversion to a pathogenic strain, respectively.

18 Further attenuation of the LAIV could generally improve its safety profile, which might come 19 at the cost of reduced immunogenicity. To solve this dilemma, we took advantage of a 20 recently defined mechanism of ER stress induction by modifying IAV non-structural protein 1

21 (NS1). The modified LAIV (AAmut/PR8) showed stronger ER stress activation in vitro and replicated to lower titers in vivo compared to its parental strain, without affecting protection against homo-subtypic or hetero-subtypic IAV strains. AAmut/PR8 could pose as a suitable 
strategy to attend the gap to the current LAIV recommendation guidelines in susceptible target populations.

\section{Introduction}

Influenza A viruses (IAV) are the etiological agents causing one of the most significant

respiratory tract infections. Hospitalizations and deaths occur mainly among high-risk groups,

which include pregnant women, children under 5-year-old, elderly and immunocompromised

patients. In the context of the recent pandemic, influenza related hospitalizations and deaths

have been lower than previous years ${ }^{1-3}$, most likely due to preventive measures widely adopted such as hand washing, sanitizing, keeping distances and providing better air

ventilation. However, seasonal IAV infections are still an important threat to healthcare

systems as a recent metadata analysis showed $7 \%$ of total acute lower respiratory infection

cases in children under 5 years were attributed to IAV ${ }^{4}$. Additionally, an out-of-season

resurgence in flu infections is expected, as the unexposed population grows wider during the pandemic ${ }^{5}$

One of the most effective ways to prevent influenza diseases is vaccination. Three types of vaccines are available for IAV: recombinant influenza vaccines or split-virus inactivated influenza vaccine (IIV), both injected intra-muscularly and a live-attenuated influenza vaccine (LAIV), in the form of a nasal spray. The LAIV uses the genetic backbone of a temperature sensitive (ts), cold-adapted ( $c a)$, attenuated (att) influenza strain derived from A/Ann

44 Arbor/6/1960 restricting vaccine virus replication to the upper respiratory tract at $33^{\circ} \mathrm{C}$, thus preventing disease onset. Additionally, LAIV mimicks a natural infection, which brings the advantage of triggering mucosal immune responses ${ }^{6}$. Hence, the general vaccine 
inactivated vaccine ${ }^{7,8}$. Adversely, its safety profile excludes vaccination of high-risk patients.

Current guidelines advise against the use in immunocompromised patients, pregnant women, adults over 50 years old and infants under 2 years old. In fact, higher rates of fever, rhinorrhea and wheezing after LAIV administration were shown in the latter population ${ }^{9,10}$. Therefore, different strategies have been explored to improve the safety of the LAIV with a large majority of them targeting the IAV non-structural protein 1 (NS1) $)^{11-15}$.

54

NS1 is a virulence factor that modulates the cellular anti-viral response ${ }^{16,17}$ and interferes with host mRNA maturation by interacting with the $30 \mathrm{kDa}$ subunit of the cleavage and polyadenylation specificity factor (CPSF30) ${ }^{18,19}$. CPSF30 is a key factor in the poly-adenylation complex and in its absence, pre-mRNAs are not polyadenylated and thus rapidly degraded, leading to a reduced host cell protein production ${ }^{20}$. The interaction between NS1 and CPSF30 is well described in the literature with a crystal structure showing the amino acids responsible for this interaction. While positions 103 and 106 are important to stabilize the interaction ${ }^{21,22}$, positions 108, 125 and 189 were shown to antagonize the innate immune response by reducing antiviral genes expression ${ }^{23}$.

We recently demonstrated that CPSF30 binding by NS1 is essential for blocking the unfolded protein response $(U P R)^{24}$. Importantly, the UPR contributes to the adjuvant effect of the licensed adjuvant $\mathrm{AS}^{25} 3^{25}$, which is a component of inactivated influenza vaccine Prepandrix. In our study, we proposed a targeted approach to mutate NS1 from the LAIV backbone and render it unable to bind CPSF30. We hypothesized that by modulating LAIV's capacity of inducing a host response, we could improve its safety profile with more efficient viral clearance and limited viral replication in the host, whilst maintaining its ability to induce a protective immune response from the activation of ER stress and UPR pathway. If successful, 
this approach could widen the patient spectrum in which a live attenuated vaccine could be applied.

\section{Results}

Abrogation of CPSF3O binding does not diminish production of LAIV in ovo or in MDCK cells

77 Deletion of the expression of the non-structural protein 1 (NS1) from LAIV has been shown to increase its attenuated phenotype ${ }^{13,15}$. We attempted a targeted approach to attenuate the LAIV by abolishing the ability of NS1 to interact with CPSF30. We used targeted mutagenesis to substitute F103S, M106I, K124E, D144R and D189G from the LAIV ca backbone and rescued the mutant virus (AAmut/PR8) by reverse genetics. The NS1 mutant virus and its parental counterpart (AAwt/PR8) are composed of the six gene segments of the cold-adapted influenza A/Ann Arbor/6/1960 plus the segments of hemagglutinin and neuraminidase of influenza A/Puerto Rico/8/1934 (PR8) and their only difference is in its NS1 gene sequence. Both viruses were grown in embryonated chicken eggs at $33^{\circ} \mathrm{C}$ and sequenced to confirm the presence of the mutations. To confirm that the five aa mutations introduced abrogate the NS1-CPSF30 binding, we overexpressed a flag-tagged CPSF30 protein in HEK 293T cells and superinfected those cells with AAmut/PR8 or AAwt/PR8. Following co-immunoprecipitation, AAwt/PR8 NS1 was able to bind CPSF30 while AAmut/PR8 NS1 showed only residual binding (Figure 1a).

Enhanced attenuation of LAIV could result in deleterious replication defects, with unwanted consequences for large scale vaccine production. We thus compared the replication of AAmut/PR8 and AAwt/PR8 in two relevant production models, embryonated chicken eggs and MDCK cells. Titers of AAwt/PR8 and AAmut/PR8 vaccine stock were comparable in both production systems (Figure 1b, c). 
binding without affecting the potential for vaccine production.

98

\section{AAmut/PR8 is attenuated without compromising protection in an adult mouse model}

In order to demonstrate that our strategy results in an attenuated phenotype in vivo, we first compared the replication of AAmut/PR8 and AAwt/PR8 in the upper and lower respiratory tract of adult mice. At 4 days post-vaccination we consistently detected AAwt/PR8 virus in snouts and lungs, while AAmut/PR8 was absent in all snout samples and present in only two lung samples at lower viral loads compared to AAwt/PR8 (Figure 2a, b). Since AAmut/PR8 displayed a clear replication defect, we asked, if the protection elicited by AAmut/PR8 was compromised compared to AAwt/PR8. 8-weeks old mice were vaccinated intranasally with $10^{2}-10^{4} \mathrm{pfu}$. The highest dose corresponds approximately to the recommended weight adjusted dose of quadrivalent LAIV in human adults ${ }^{26}$. We challenged the animals at 21 days post-vaccination with $10 \mathrm{LD}_{50}$ of influenza $A /$ Netherlands/602/2009 (H1N1) strain (homosubtypic/heterologous challenge), simulating a severely drifted virus. We monitored body weight (Figure 2c) and survival (Figure $2 \mathrm{~d}$ ) for 14 days. All animals were protected after either vaccine (Figure $2 \mathrm{~d}$ ) when using $10^{4} \mathrm{pfu}$. While we found a trend towards reduced protection with lower doses of AAmut/PR8 (Figure S1a, b), there was overall no statistically significant difference observed for the protective dose $50\left(\mathrm{PD}_{50}\right)$ calculated for the dose response to both vaccines (Figure 2e). We also showed that neither vaccine caused any alterations in body weight of adult mice (Figure 2f), reflecting the already attenuated phenotype of this virus. In conclusion, compared to AAwt/PR8, our vaccine candidate AAmut/PR8 had lower replication in adult's mice respiratory tract, while conferring full protection against a homosubtypic/heterologous challenge. 


\section{AAmut/PR8 is attenuated without compromising protection in neonatal mice}

122 Younger children were reported to show adverse effects, such as wheezing after vaccination ${ }^{9}$.

123 We postulated that by showing lower replication, our vaccine candidate has a higher

124 attenuation degree, which could ameliorate the safety concerns regarding its use in infants

125 younger than 2 years. Thus, we characterized AAwt/PR8 and AAmut/PR8 replication in a more

126 appropriate model using 7-days old mice. We vaccinated neonatal mice via the intranasal

127 route and determined vaccine viral loads after 2, 4 and 6 days. In accordance with the results

128 obtained in adult mice, at 2 days post-vaccination we observed lower titers of AAmut/PR8

129 compared to AAwt/PR8 in snouts (Figure 3a). While at day 4 both vaccine viral loads were

130 similar and reduced compared to day 2, we observed that on day 6 post-vaccination,

131 AAwt/PR8 virus titers were dispersed with some animals presenting high titers while others

132 had non-detectable virus. This was not the case for the AAmut/PR8 group, which at day 6

133 post-vaccination had either low viral titers or non-detectable virus. In the lungs, we did not

134 consistently detect virus after infection with either vaccine, which might be a consequence of

135 vaccine administration in absence of anesthesia. We conclude from these data that

136 AAmut/PR8 is cleared faster from the airways of neonatal mice compared to AAwt/PR8.

137 It is well known that the immune system of young children is in an immature state with

138 compromised immune responses to vaccines ${ }^{27,28}$. Thus, we asked if our attenuation

139 phenotype would have an impact on the protection elicited by this vaccine in our neonatal

140 model. To answer this question, we evaluated both short-term protection after a

141 heterosubtypic challenge and long-term protection after a homosubtypic/heterologous

142 challenge. Neonatal mice were vaccinated with increasing doses of each vaccine and

143 challenged with either influenza A/Vietnam/2004 after 21 days or A/Netherlands/09 after 49 
days. In the heterosubtypic challenge, significant protection was observed in all doses for both vaccines in a similar fashion (Figure 3b,c and Figure S2a-b). Interestingly, in the long-

146 term homosubtypic/heterologous challenge, while the middle dose of AAmut/PR8 was not

147 protective compared to AAwt/PR8 (Figure S2c-d), both vaccines were similarly protective at

148 the higher dose of $10^{4} \mathrm{PFU}$ (Figure 3d,e). Additionally, there was no overall statistically

149 significant difference observed for the protective dose 50 (PD50) of each vaccine (Figure 3f).

150 We also showed that neither vaccine caused any reduction in body weight gain of neonatal

151 mice (Figure 3g), once again reflecting the already attenuated phenotype of this virus. In

152 conclusion, compared to AAwt/PR8, our vaccine candidate AAmut/PR8 is mildly attenuated

153 in neonatal mice, while still able to protect against a long-term homosubtypic challenge and

154 a heterosubtypic challenge.

155

AAwt/PR8 and AAmut/PR8 induces overall comparable innate immune responses in neonatal

mice snouts

Blocking of CPSF30 by NS1 is a cornerstone in the host protein synthesis shutoff initiated by

159 IAV infection. This broadly active strategy limits the innate antiviral response ${ }^{29}$. To further explore the safety profile of AAmut/PR8, we looked into the innate response induced by both

161 vaccines in neonatal mice snouts at 2 days post-vaccination, when vaccine loads were highest. Both vaccines induced antiviral cytokines $\mathrm{Cx} / 10$ and $\mathrm{C} / 5$ expression at similar levels

163 indicating similar innate responses. When we looked into pro-inflammatory cytokines, no significant expression of Tnfa and $/ / 6$ was observed compared to mock-vaccinated animals responses as a reason for the enhanced attenuation while maintaining immunogenicity. 
Remarkably, $/ 16$ expression was suppressed in AAwt/PR8 vaccinated animals compared to

AAmut/PR8 (figure 4). The same NS1 mutations introduced in AAmut/PR8 NS1 were described to modulate ER stress activation and UPR through the XBP1 pathway ${ }^{24}$ which can lead to IL-6 expression ${ }^{30,31}$. Therefore, to explore this pathway as a mechanism of attenuation

171 we evaluated the expression of two target genes regulated by the UPR. Neither AAmut/PR8 nor AAwt/PR8 vaccinated neonatal and adult mice induced significant expression of Dnajb9

173 (ERdj4) or Ddit3 (CHOP) compared to mock-vaccinated animals (Figure S3). UPR is notoriously difficult to measure in vivo due to its transient activation and a poor signal-to-noise ratio from a low percentage of infected cells in the upper respiratory tract (refs). Thus, we decided to characterize AAmut/PR8 ability to modulate ER stress activation using relevant in vitro cell models.

NS1 binding to CPSF30 was previously shown to modulate the induction of the UPR ${ }^{24}$.

181 Therefore, we asked if the mutation in AAmut/PR8 NS1 allowed the establishment of the UPR upon infection. We infected A549 cells and evaluated XBP1 splicing and sXBP1 protein expression levels by western blot. Our results show an increase in the spliced form of XBP1 protein levels in AAmut/PR8-infected A549 as compared to AAwt/PR8 (Figure 5a). In addition, the spliced form of XBP1 mRNA amount observed by semi-quantitative RT-PCR matched with the increased amount of spliced XBP1 protein (Figure 5b). We also analysed by RT-qPCR the induction of genes downstream of the transcription factor SXBP1, namely DDIT3 and DNAJB9. Compared to AAwt/PR8, the expression of these two genes was increased in cells infected with AAmut/PR8 (Figure 5c, upper panel), in line with previous published results. This increase 
$1915 c$, lower panel) indicating that this phenomenon is species independent and reflects a

192 general phenotype during viral replication, when the ability of NS1 to bind CPSF30 is

193 abrogated. Also, these observations were not explained by a difference in replication of

194 AAmut/PR8 since viral replication was comparable in both cell models (Figure $5 \mathrm{~d}$ ).

195 Lastly, we took advantage of a more relevant model using stratified primary human nasal

196 epithelial cells grown in air-liquid interface culture. These cells would be the first

197 encountering the LAIV virus in case of vaccination of human patients. In this model, we also

198 observed induction of both UPR-induced genes and no differences in the induction of antiviral

199 response genes (Figure 5e).

200 Overall, our results show that AAwt/PR8 and AAmut/PR8 mount a comparable antiviral

201 response in relevant cell models and in vivo, which do not explain the reduced in vivo replication and comparable immunogenicity. In contrast, AAmut/PR8 allows the establishment of a robust UPR compared to AAwt/PR8 in vitro, which might contribute to the enhanced attenuated profile of AAmut/PR8 observed in vivo. 


\section{Discussion:}

207 LAIV is currently only approved for use in target population ranging from healthy 2 years-old to 49 years-old. Thus key-populations, such as pregnant women, elderly subjects, children under 2 years-old and immunocompromised patients do not benefit from the advantages of

210 this vaccine option, such as a wider immune response and avoidance of traumatic injections

211 specially in paediatrics. The rationale behind this limitation is the fear of reversion to a

212 virulent strain that could cause disease based on experience with other replicating vaccines

213 such as measles-mumps-rubella (MMR), yellow fever and oral polio, which have shown

214 reported cases of uncontrolled replication and were able to cause disease in immunocompromised patients ${ }^{32-35}$. Not surprisingly, several attempts have tried to improve LAIV safety, including approaches that either delete the NS1 gene of LAIV or mutate it, rendering a more attenuated virus ${ }^{11-15}$. In fact, two clinical trials tested NS1-deleted-NS1 LAIVs containing $H A$ and NA from influenza virus $H 1 N 1, H 3 N 2$, and $B$ strains in adult volonteers. As for the parental LAIV a single intranasal dose was well tolerated, safe, not shedded from the patient and induced significant levels of strain-specific and crossneutralizing antibodies in vaccinated humans ${ }^{36,37}$. However, it remains currently unclear, if a

222 similar safety profile could be achieved in more susceptible patient groups.

223 Another major concern in administering LAIV in children under 2 years-old is the higher

224 incidence of side effects observed such as wheezing, cough and runny nose. Although there

225 is a lack of evidence pinpointing to the mechanism responsible for this, an association between vaccine virus shedding and runny nose after vaccination in this target population

227 has been described ${ }^{38}$. We showed here lower viral replication and earlier clearance of a CPSF30 binding mutant LAIV in neonatal mice, which hints to a generally safer profile in children under 2 years old. 
The innate immune response to a replicating virus contributes to the onset of the symptoms associated with LAIV administration in this population. Several cytokines and chemokines have been associated to lung damage during acute influenza infection. Indeed, CXCL-10, RANTES, IL-6 and TNF-a are all involved in the host response to IAV and can lead to a 234 detrimental response $\mathrm{S}^{39,40}$. A potential danger of eliminating the antagonism of CPSF30 in LAIV could have been an exaggerated inflammatory response. However, in our in vivo models, AAmut/PR8 induced similar levels of pro-inflammatory cytokines as AAwt/PR8 suggesting that the attenuated phenotype observed does not exacerbate the host immune response to the vaccine.

Curiously, we observed a slightly lower expression of II-6 in AAwt/PR8 vaccinated neonatal mice (Figure 4). The pathophysiological role of this cytokine is still unclear, with reports showing a correlation between its expression and clinical score symptoms in human patients ${ }^{39,41}$ while others report a protective role for this cytokine during influenza infection in mice ${ }^{42-44}$. Moreover, IL-6 has been described as a target gene of the UPR transcription

244 factor sXBP1 during ER stress activation $30,45,46$. In vitro, AAmut/PR8 more potently induces ER 245 stress activation and UPR in accordance with our previous work ${ }^{24}$. Unfortunately, we could not demonstrate this pathway's activation in vivo most likely due to the sensitivity of the

247 techniques used. The lack of appropriate models such as IRE1 $\alpha$ or XBP1 knockout mice also renders it difficult to assess ER stress activation impacts in vivo.

249 Notwithstanding, together with the fact that AAmut/PR8 is attenuated and still able to protect both adult and neonatal mice against a homosubtypic and heterosubtypic challenge,

251 we propose that ER stress activation during AAmut/PR8 administration compensates for its lower replication and maintains this vaccine immunogenicity and efficacy. This hypothesis is

253 further corroborated by the fact that ER stress has been directly implicated in the 
establishment of an immune response to influenza vaccines as a mechanism of action of the adjuvant $\mathrm{ASO3}^{25}$.

256 The work shown here characterizes for the first time the LAIV virus in a neonatal mice model and presents an alternative vaccine candidate to LAIV with an even more pronounced attenuated phenotype while still able to maintain its protection. We confirm that this vaccine is still scalable and able to be produced at similar titers as the current vaccine virus used in Fluenz ${ }^{\circledR}$ Tetra to attend a population-wise demand and presents a safer option to the current LAIV with the possibility of being administered to target populations where uncontrolled replication of the virus is a serious concern.

References:

1. Feng, L. et al. Impact of COVID-19 outbreaks and interventions on influenza in China and the United States. Nat. Commun. 12, 1-8 (2021).

2. Public Health England. Weekly national Influenza and COVID-19 surveillance report Executive summary - Week 29 2021. (2021). https://www.cdc.gov/flu/weekly/weeklyarchives2020-2021/week20.htm. influenza in children under 5 years in 2018: a systematic review and modelling study. Lancet. Glob. Heal. 8, e497-e510 (2020). 
278

279

280

281

6. Jang, Y. H. \& Seong, B. L. Immune responses elicited by live attenuated influenza vaccines as correlates of universal protection against influenza viruses. Vaccines $\mathbf{9}$, (2021).

7. Pebody, R., McMenamin, J. \& Nohynek, H. Live attenuated influenza vaccine (LAIV): Recent effectiveness results from the USA and implications for LAIV programmes elsewhere. Arch. Dis. Child. 103, 101-105 (2018).

8. Hoft, D. F. et al. Live and inactivated influenza vaccines induce similar humoral responses, but only live vaccines induce diverse T-cell responses in young children. J. Infect. Dis. 204, 845-853 (2011).

9. Prutsky, G. J., Domecq, J. P., Elraiyah, T., Prokop, L. J. \& Murad, M. H. Assessing the evidence: Live attenuated influenza vaccine in children younger than 2 years. A systematic review. Pediatr. Infect. Dis. J. 33, 106-115 (2014).

10. Belshe, R. B. et al. Live Attenuated versus Inactivated Influenza Vaccine in Infants and Young Children. N. Engl. J. Med. 356, 685-696 (2007).

11. Pica, N., Langlois, R. A., Krammer, F., Margine, I. \& Palese, P. NS1-Truncated Live Attenuated Virus Vaccine Provides Robust Protection to Aged Mice from Viral Challenge. J. Virol. 86, 10293-10301 (2012).

12. Ngunjiri, J. M., Ali, A., Boyaka, P., Marcus, P. I. \& Lee, C. W. In vivo assessment of NS1truncated influenza virus with a novel SLSYSINWRH motif as a self-adjuvanting live attenuated vaccine. PLoS One 10, 1-16 (2015).

13. Wang, P. et al. Generation of DeLNS1 influenza viruses: A strategy for optimizing live attenuated influenza vaccines. MBio 10, (2019).

14. Hilimire, T. A., Nogales, A., Chiem, K., Ortego, J. \& Martinez-sobrido, L. Increasing the safety profile of the master donor live attenuated influenza vaccine. Pathogens $\mathbf{9}$, 
(2020).

15. Richt, J. A. \& García-Sastre, A. Attenuated Influenza Virus Vaccines with Modified NS1 Proteins. Curr. Top. Microbiol. Immunol. 333, 177-195 (2009).

16. Hale, B. G., Randall, R. E., Ortin, J. \& Jackson, D. The multifunctional NS1 protein of influenza A viruses. J. Gen. Virol. 89, 2359-2376 (2008).

17. Das, K. et al. Structural basis for suppression of a host antiviral response by influenza a virus. Proc. Natl. Acad. Sci. U. S. A. 105, 13093-13098 (2008).

18. Nemeroff, M. E., Barabino, S. M. L., Li, Y., Keller, W. \& Krug, R. M. Influenza virus NS1 protein interacts with the cellular 30 kDa subunit of CPSF and inhibits $3^{\prime}$ end formation of cellular pre-mRNAs. Mol. Cell 1, 991-1000 (1998).

19. Twu, K. Y., Noah, D. L., Rao, P., Kuo, R.-L. \& Krug, R. M. The CPSF30 Binding Site on the NS1A Protein of Influenza A Virus Is a Potential Antiviral Target. J. Virol. 80, 3957-3965 (2006).

20. Sun, Y. et al. Molecular basis for the recognition of the human AAUAAA polyadenylation signal. Proc. Natl. Acad. Sci. U. S. A. 115, E1419-E1428 (2018).

21. Wang, B. X., Brown, E. G. \& Fish, E. N. Residues F103 and M106 within the influenza A virus NS1 CPSF4-binding region regulate interferon-stimulated gene translation initiation. Virology 508, 170-179 (2017).

22. Kochs, G., García-Sastre, A. \& Martínez-Sobrido, L. Multiple Anti-Interferon Actions of the Influenza A Virus NS1 Protein. J. Virol. 81, 7011-7021 (2007).

23. Hale, B. G. et al. Inefficient Control of Host Gene Expression by the 2009 Pandemic H1N1 Influenza A Virus NS1 Protein. J. Virol. 84, 6909-6922 (2010). synthesis shutoff. Proc. Natl. Acad. Sci. U. S. A. 118, (2021). 
25. Givord, C. et al. Activation of the endoplasmic reticulum stress sensor IRE1 $\alpha$ by the vaccine adjuvant $\mathrm{ASO}$ contributes to its immunostimulatory properties. npj Vaccines 3, (2018).

26. Fluenz Tetra nasal spray suspension Influenza vaccine (live attenuated, nasal) $\begin{array}{lllll}\text { Summary of } & \text { Product } & \text { Characteristics } & \text { (SmPC) }\end{array}$ https://www.medicines.org.uk/emc/medicine/29112\#gref.

27. Siegrist, C. A. Neonatal and early life vaccinology. Vaccine 19, 3331-3346 (2001).

28. Prabhudas, M. et al. Challenges in infant immunity: Implications for responses to infection and vaccines. Nat. Immunol. 12, 189-194 (2011).

29. Noah, D. L., Twu, K. Y. \& Krug, R. M. Cellular antiviral responses against influenza A virus are countered at the posttranscriptional level by the viral NS1A protein via its binding to a cellular protein required for the $3^{\prime}$ end processing of cellular pre-mRNAS. Virology 307, 386-395 (2003).

30. Martinon, F., Chen, X., Lee, A.-H. \& Glimcher, L. H. TLR activation of the transcription factor XBP1 regulates innate immune responses in macrophages. Nat. Immunol. 2010 115 11, 411-418 (2010).

31. Sanchez, C. L., Sims, S. G., Nowery, J. D. \& Meares, G. P. Endoplasmic reticulum stress differentially modulates the IL- 6 family of cytokines in murine astrocytes and macrophages. Sci. Rep. 9, 1-12 (2019).

32. Measles Pneumonitis Following Measles-Mumps-Rubella Vaccination of a Patient with HIV Infection, 1993. https://www.cdc.gov/mmwr/preview/mmwrhtml/00043110.htm.

33. Kengsakul, K., Sathirapongsasuti, K. \& Punyagupta, S. Fatal myeloencephalitis following yellow fever vaccination in a case with HIV infection. J. Med. Assoc. Thai. 85, 131-4 
(2002).

351

352

353

34. Adverse Events Associated with Childhood Vaccines. (National Academies Press, 1994). doi:10.17226/2138.

35. Macklin, G. Update on Immunodeficiency-Associated Vaccine-Derived Polioviruses Worldwide, July 2018-December 2019. MMWR. Morb. Mortal. Wkly. Rep. 69, 913-917 (2020).

36. Mössler, C. et al. Phase I/II trial of a replication-deficient trivalent influenza virus vaccine lacking NS1. Vaccine 31, 6194-6200 (2013).

37. Wacheck, V. et al. A novel type of influenza vaccine: Safety and immunogenicity of replication-deficient influenza virus created by deletion of the interferon antagonist NS1. J. Infect. Dis. 201, 354-362 (2010).

38. Mallory, R. M., Yi, T. \& Ambrose, C. S. Shedding of Ann Arbor strain live attenuated influenza vaccine virus in children 6-59 months of age. Vaccine $\mathbf{2 9}, 4322-4327$ (2011).

39. Hayden, F. G. et al. Local and systemic cytokine responses during experimental human influenza A virus infection. Relation to symptom formation and host defense. J. Clin. Invest. 101, 643-649 (1998).

40. De Jong, M. D. et al. Fatal outcome of human influenza A (H5N1) is associated with high viral load and hypercytokinemia. Nat. Med. 12, 1203-1207 (2006).

41. Kaiser, L., Fritz, R. S., Straus, S. E., Gubareva, L. \& Hayden, F. G. Symptom pathogenesis during acute influenza: Interleukin-6 and other cytokine responses. J. Med. Virol. 64, $262-268$ (2001).

42. Lauder, S. N. et al. Interleukin-6 limits influenza-induced inflammation and protects against fatal lung pathology. Eur. J. Immunol. 43, 2613-2625 (2013).

43. Yang, M. L. et al. IL-6 ameliorates acute lung injury in influenza virus infection. Sci. Rep. 
7, 1-11 (2017).

375 44. Bonney, E. A. et al. Protective Intranasal Immunization Against Influenza Virus in Infant Mice Is Dependent on IL-6. Front. Immunol. 11, 1-13 (2020).

377 45. Bud Nicola, and C. K. N. J.-E. K. V. B. V. E. T. R. M. Emerging functions of the unfolded protein response in immunity. Nat. Immunol. 910-919 (2014) doi:doi:10.1038/ni.2991.

379 46. Flores-Santibáñez, F., Medel, B., Bernales, J. I. \& Osorio, F. Understanding the Role of 380 the Unfolded Protein Response Sensor IRE1 in the Biology of Antigen Presenting Cells. Cells 8, (2019). 


\section{FIG 1}
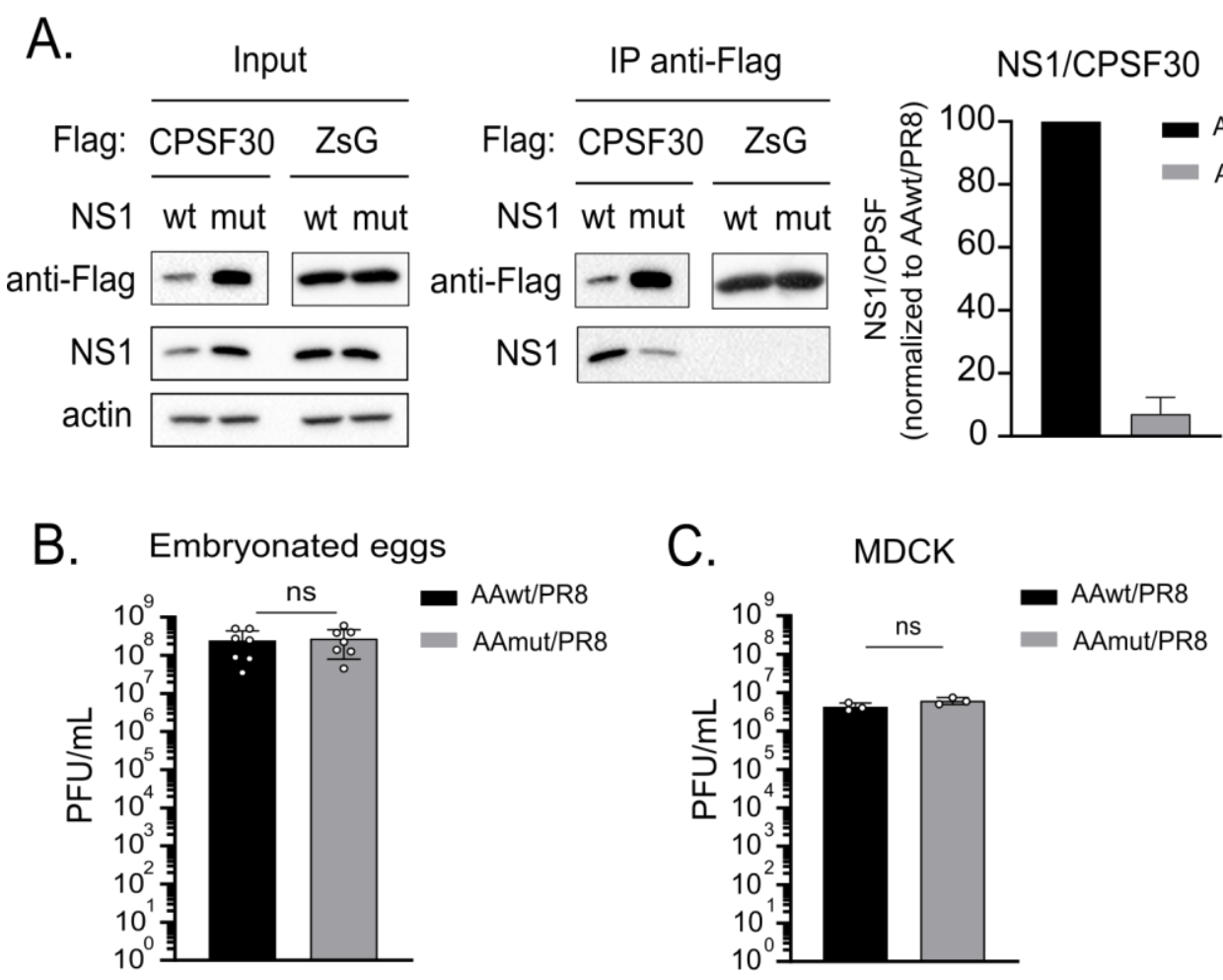

Figure 1: AAmut/PR8 NS1 binds to CPSF30 without compromising vaccine virus growth in

\section{eggs or MDCK cells.}

(a) 293 cells were transfected with pCAGGS.Flag-CPSF30 or pCAGGS.Flag-ZsGreen for $24 \mathrm{~h}$ prior infection with either AAwt/PR8 or AAmut/PR8 at a MOI of 5. Cells were incubated for $16 \mathrm{~h}$ at $33^{\circ} \mathrm{C}$ and then lysed. Anti-Flag M2 affinity beads were used to immunoprecipitate (IP) the flag-tagged protein and its interactors. Precipitates were separated by SDS-PAGE and detected by WB using anti-flag antibody and anti-NS1 antibody and the amount of NS1 protein bound to Flag-CPSF30 was quantified using densiometric analysis $(n=2)$

( $b$ and c) Ten-days old embryonated chicken eggs (b) were infected with 100 PFU and MDCK B cells (c) were infected at a $\mathrm{MOI}$ of 0.1 . After $46 \mathrm{~h}$ at $33^{\circ} \mathrm{C}$ allantoic fluid and supernatant were collected. Viral titers were determined by plaque assay in MDCK cells at $33^{\circ} \mathrm{C}$. Graphs 
represent the mean $\pm S D$ of $n=7$ repeats in eggs or $n=3$ repeats in MDCK cells, respectively, and the statistical significances were determined using unpaired t-test. $n s=$ non-significant

\section{FIG 2}

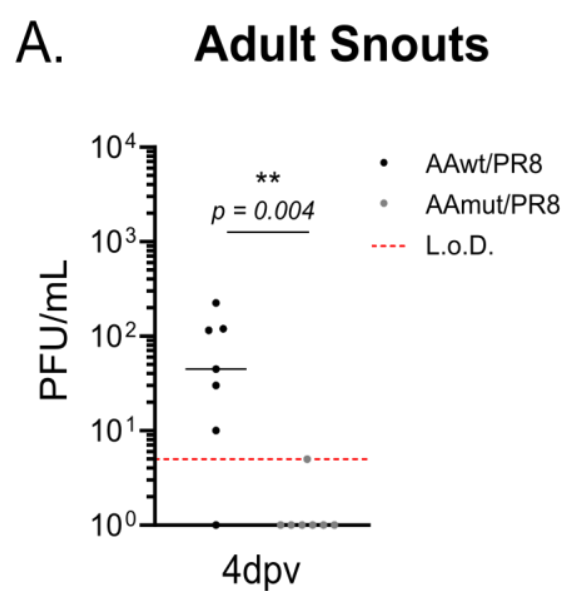

C. Homosubtypic challenge

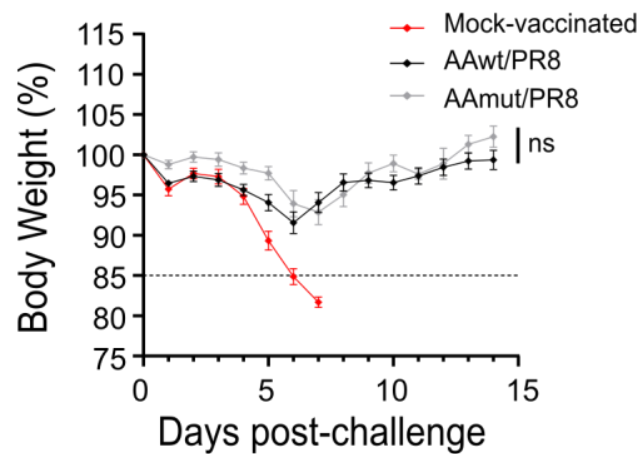

E.

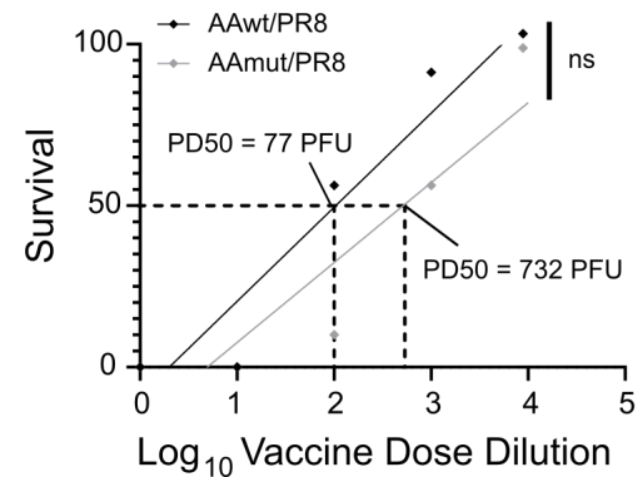

B. Adult Lungs

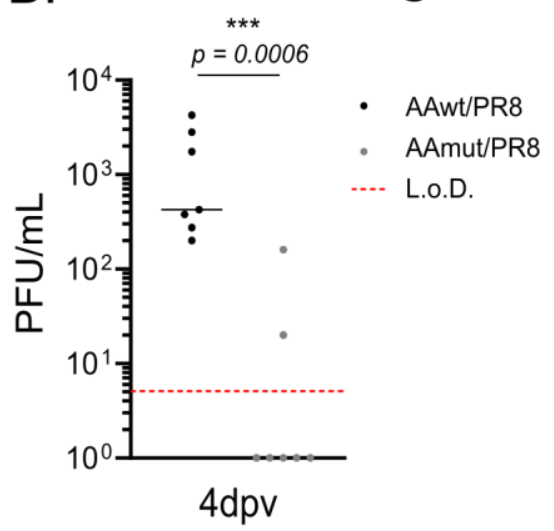

\section{Homosubtypic challenge}

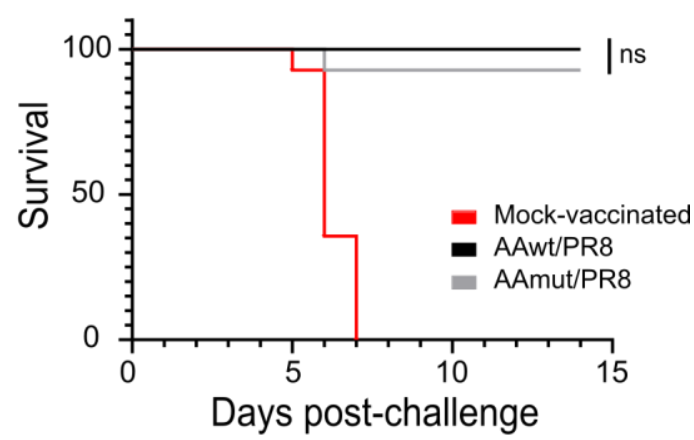

F. Vaccine susceptibility

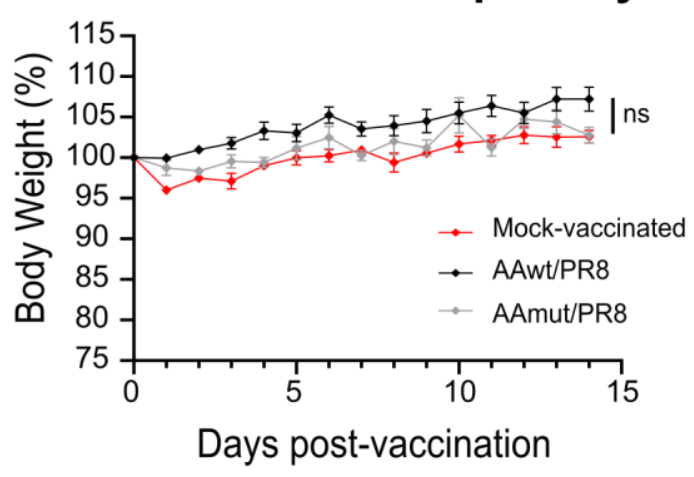

Figure 2: AAmut/PR8 is attenuated but protects adult mice against a homosubtypic/heterologous challenge. 
( $a$ and b) Female 8-weeks-old mice were vaccinated intranasally under anaesthesia with $10^{5}$ PFU AAwt/PR8 or AAmut/PR8 in $25 \mu \mathrm{L}$ PBS. At 4 days post-vaccination snouts (a) and lungs (b) were collected and vaccine viral titters were determined by plaque assay ( $n=7$ per group). (c - e) Female 8-weeks-old mice vaccinated with $10^{4}$ PFU of AAwt/PR8 or AAmut/PR8 were challenged at day 21 post-vaccination with 20 PFU $\left(10 \times L_{50}\right)$ of mouse adapted A/Netherlands/602/2009 (H1N1) in $20 \mu \mathrm{L}$ PBS under light anaesthesia ( $n=14$ per group). Body weight (c) and survival (d) were monitored for 14 days post-challenge. $\mathrm{PD}_{50}$ was calculated according to Reed \& Muench. Linear regression was used to determine statistical significance between the two $\mathrm{PD}_{50}(\mathrm{e})$.

(f) Body weight was followed for 14 days after vaccination with $10^{4} \mathrm{PFU}$ of AAwt/PR8 or AAmut/PR8. The statistical significances between AAwt/PR8 group and AAmut/PR8 group were determined using Mann-Whitney test in panels a and b; two-way ANOVA with the Geisser-Greenhouse correction and post-hoc Dunn's multiple comparisons test in panel c and $\mathrm{f}$ and Mantel-Cox test in panel d. ${ }^{*} p<0.05,{ }^{* *} p<0.01,{ }^{* * *} p<0.001$, ns $=$ non-significant, L.o.D. = limit of detection $(5 \mathrm{PFU} / \mathrm{mL})$. Graphs are representative of 3 independent experiments and indicate median for panels $a$ and $b$ or mean \pm SEM for panels $c$ and $f$. Symbols represent data from individual mice for panels a and $\mathrm{b}$. Black dotted line represents $15 \%$ body weight loss cut-off. 


\section{FIG 3}

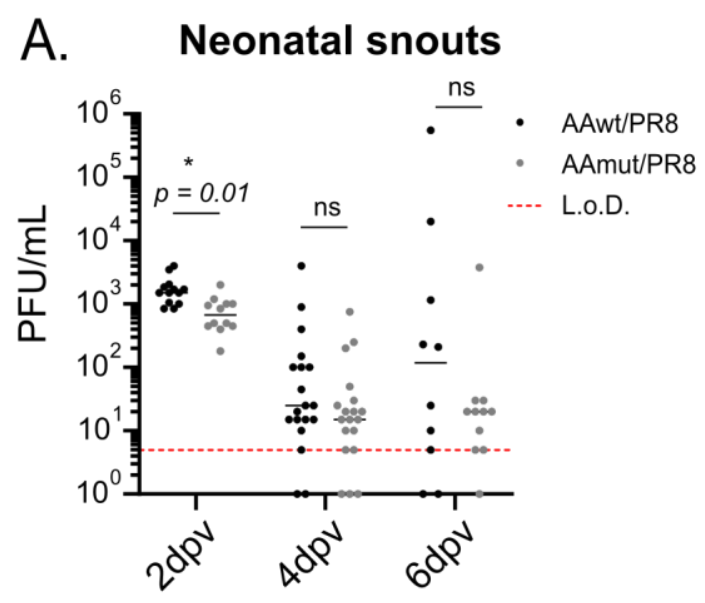

\section{B. Heterosubtypic}
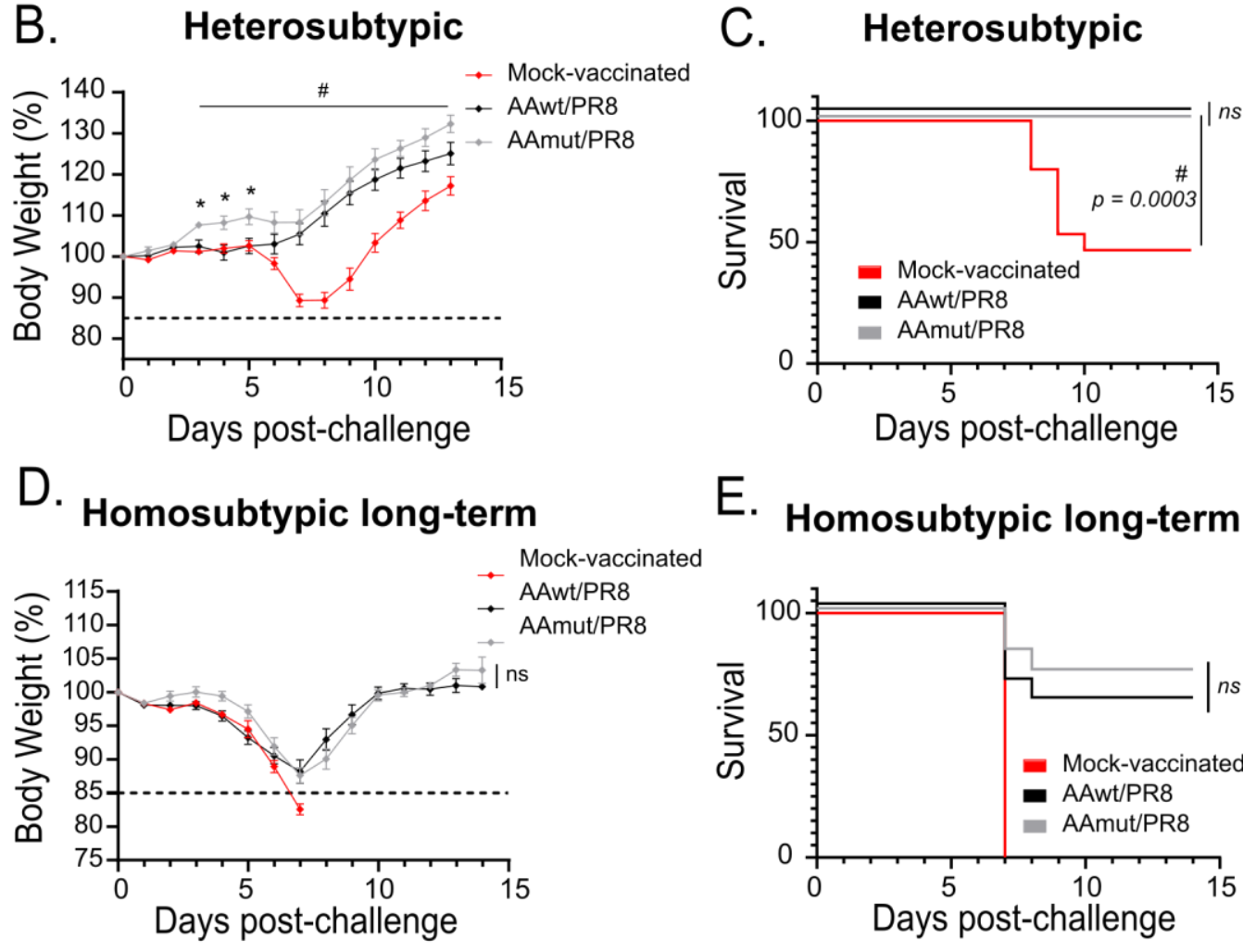

E. Homosubtypic long-term
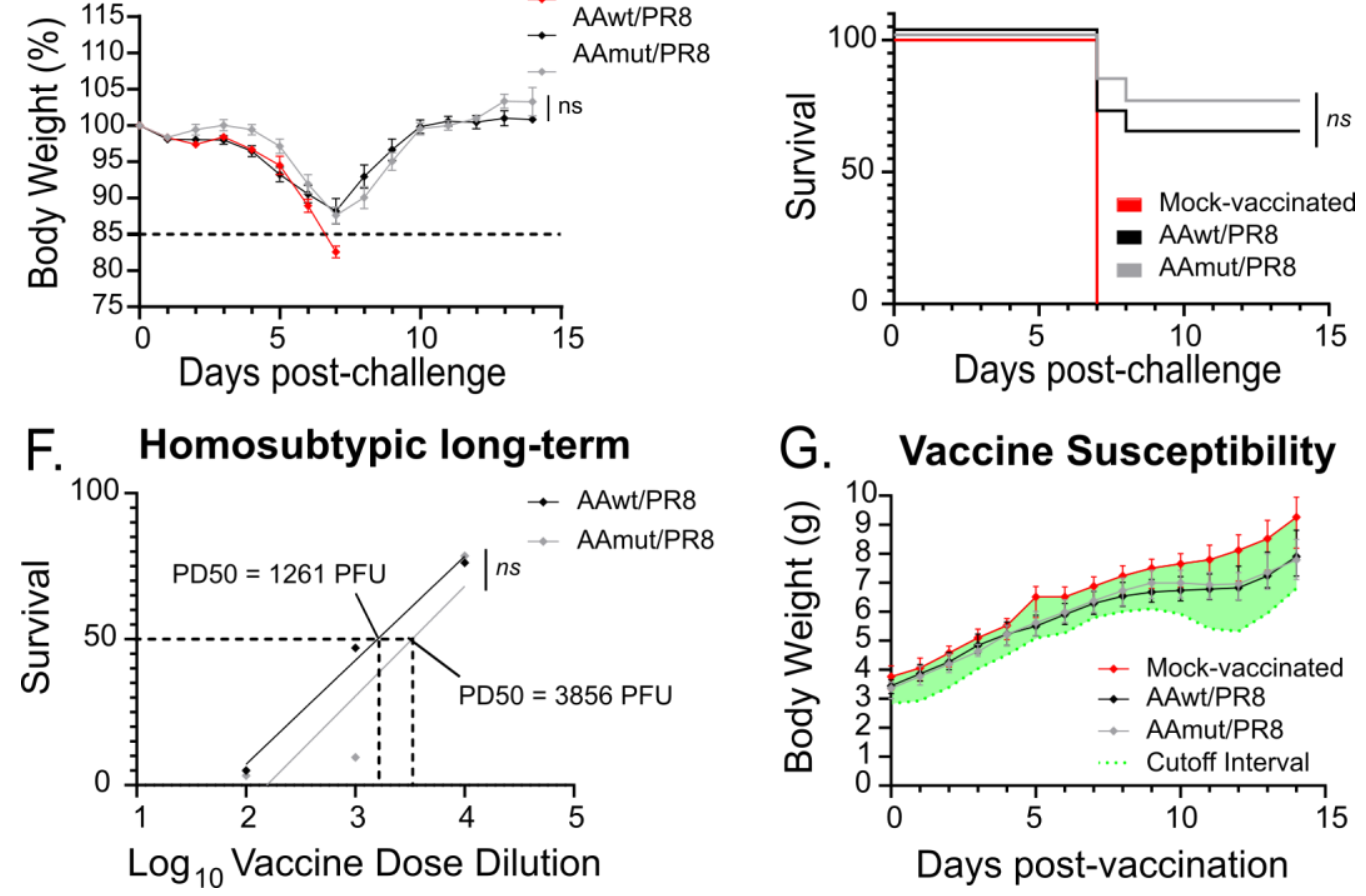

Figure 3: AAmut/PR8 is attenuated and protects neonatal mice against long-term homosubtypic/heterologous challenge and heterosubtypic challenge. 
(a) Seven-days-old mice were vaccinated intranasally with $10^{5}$ PFU of AAwt/PR8 or AAmut/PR8 in $5 \mu \mathrm{L}$ PBS. At 2, 4 and 6 days post-vaccination vaccine viral titters in the snouts were determined by plaque assay ( $n=10-19$ per group).

$(b-c)$ Vaccinated 7-days-old mice with $10^{4} \mathrm{PFU}$ of AAwt/PR8 or AAmut/PR8 were challenged at day 21 post-vaccination with $10^{3} \mathrm{PFU}\left(20 \times \mathrm{LD}_{50}\right)$ of A/Vietnam/1203/2004 (H5N1) in $20 \mu \mathrm{L}$ PBS under anaesthesia ( $n=11$ per group). Body weight (b) and survival (c) were monitored for 14 days post-challenge.

$(d-f)$ Vaccinated 7-days-old mice with $10^{4} \mathrm{PFU}$ of AAwt/PR8 or AAmut//PR8 were challenged at day 49 post-vaccination with $20 \mathrm{PFU}\left(10 \times \mathrm{LD}_{50}\right)$ of $\mathrm{A} /$ Netherlands/602/2009 (H1N1) in $20 \mu \mathrm{L}$ PBS under anaesthesia ( $n=11-17$ per group). Body weight (d) and survival (e) were monitored for 14 days post-challenge. PD $_{50}$ was calculated according to Reed \& Muench. Linear regression was used to determine statistical significance between the two $P D_{50}(f)$.

(g) Body weight was followed for 14 days after vaccination with $10^{4}$ PFU of AAwt/PR8 or AAmut/PR8 and the cut-off was defined as twice the difference between the average weight of the non-vaccinated group and the lightest mouse of that group, calculated daily.

The statistical significances between AAwt/PR8 group versus AAmut/PR8 group were determined using two-way ANOVA with the Geisser-Greenhouse correction and post-hoc Dunn's multiple comparisons test in panels $a, b$ and $d$ and Mantel-Cox test in panel $c$ and e. ${ }^{*} p<0.05,{ }^{* *} p<0.01,{ }^{* * *} p<0.001,{ }^{*} p<0.05$ between mock-vaccinated and AAmut/PR8 groups, $n s=$ non-significant, L.o.D. = limit of detection $(5 \mathrm{PFU} / \mathrm{mL})$. Graphs are representative of 2-3 independent experiments and indicate median for panel $a$, mean \pm SEM for panels $b, d$ and g. Symbols represent data from individual mice for panel a. 


\section{FIG 4}
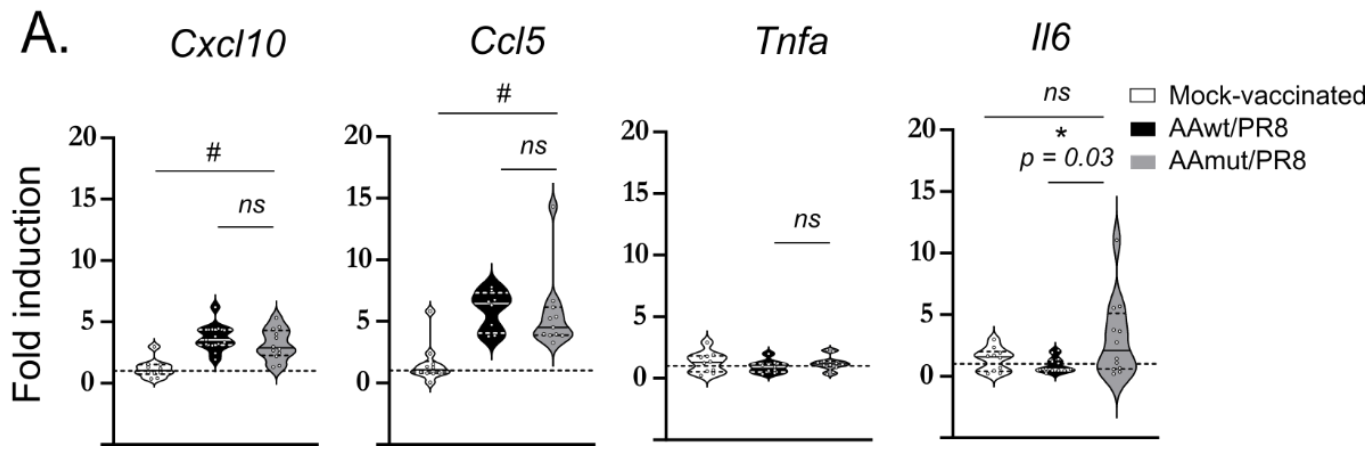

Figure 4: AAwt/PR8 and AAmut/PR8 induces overall comparable innate immune responses

in neonatal mice snouts. Seven-days-old mice $(n=11-12)$ were vaccinated intranasally with $10^{5} \mathrm{PFU}$ of AAwt/PR8 or AAmut/PR8 in $5 \mu \mathrm{L}$ PBS. At 2 days post-vaccination snouts were harvested and RT-qPCR was performed from isolated RNA. The statistical significances between groups were determined using one-way ANOVA and post-hoc Tukey's multiple comparisons test. ${ }^{*} p<0.05,{ }^{* *} p<0.01,{ }^{* * *} p<0.001,{ }^{*} p<0.05$ ns $=$ non-significant. Graphs are representative of 2 independent experiments and median is represented. 


\section{FIG 5}

A.

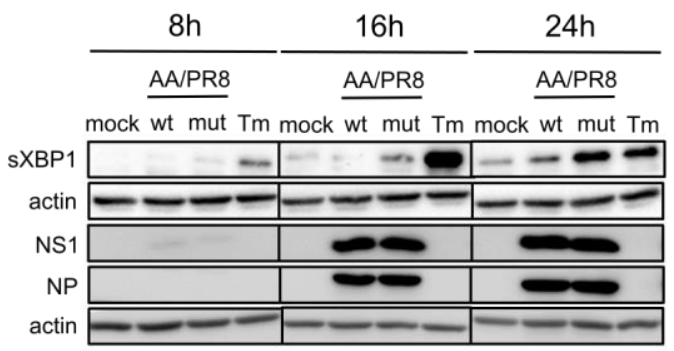

B.

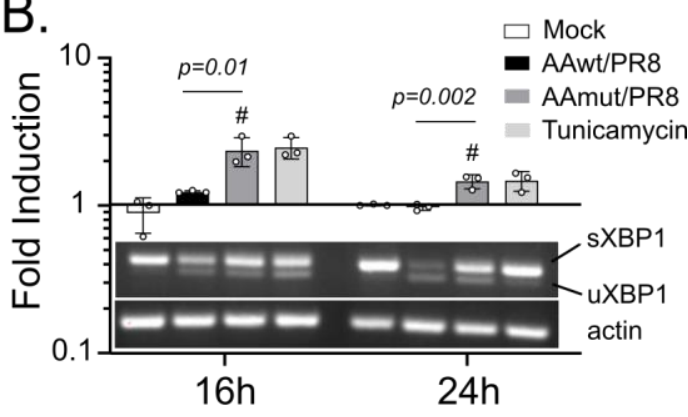

D.

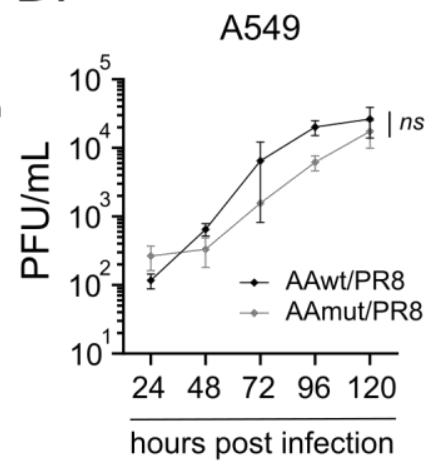

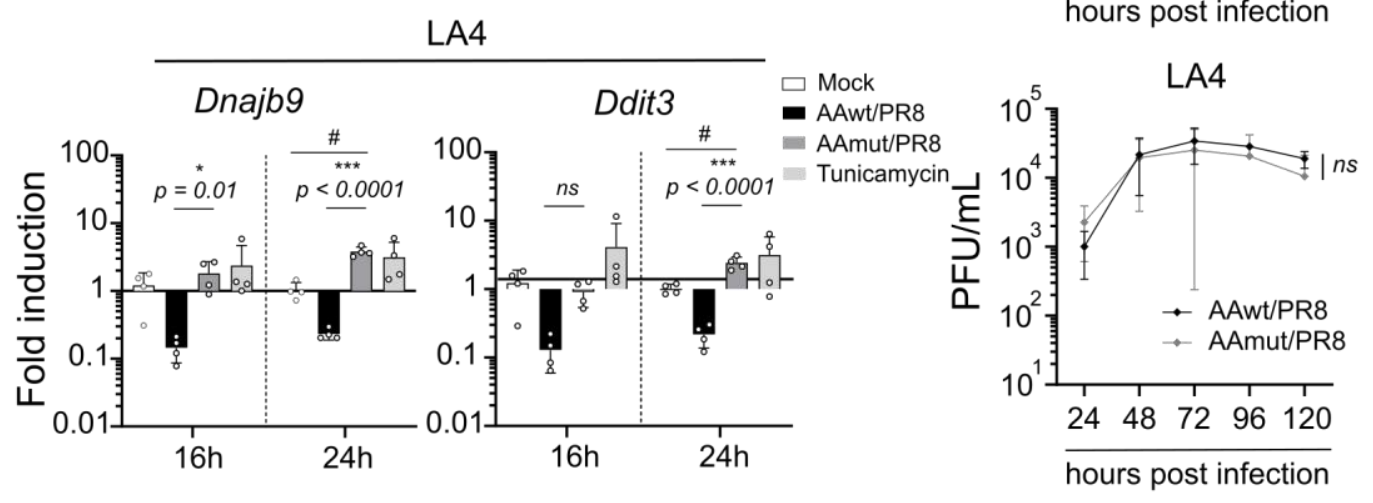

E.
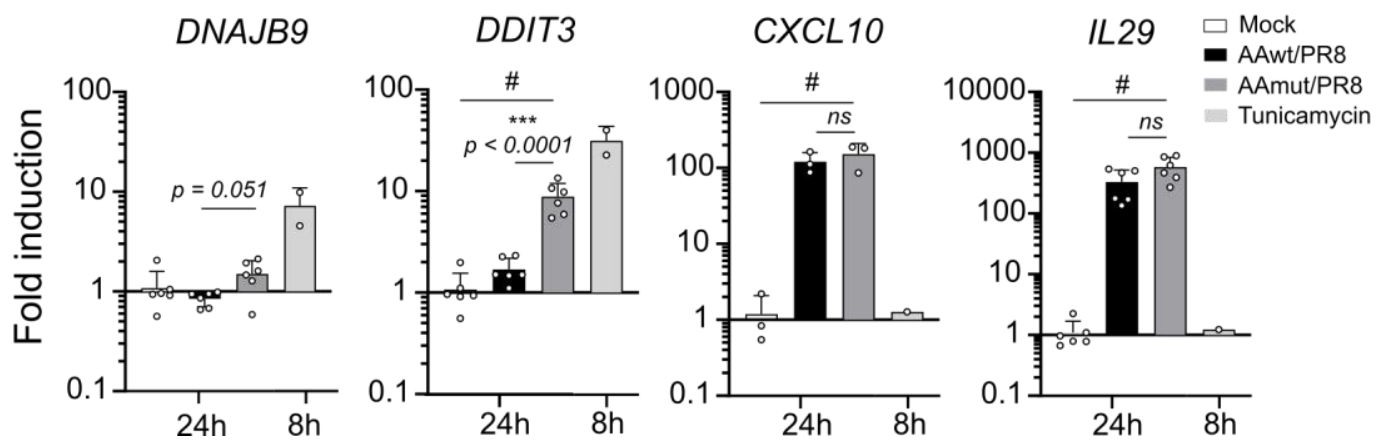

Figure 5: AAmut/PR8 NS1 allows unfolded protein response activation in murine and

\section{human cell models.}


(a - b) A549 cells were infected at a MOI of 5 with AAwt/PR8 or AAmut/PR8 or treated with tunicamycin at $5 \mathrm{ug} / \mathrm{ml}(\mathrm{n}=3)$. Cells were collected at 8,16 and $24 \mathrm{~h}$ post infection and lysates were analysed by western blot for sXBP1, actin, NP and NS1 protein levels (a). At $16 \mathrm{~h}$ and 24 h post infection RT-PCR was performed from isolated RNA for the spliced form of XBP1 mRNA (b). Graph represents fold induction of sXNP mRNA.

(c) A549 cells (upper panel) or LA4 cells (lower panel) were infected at a MOI of 5 with AAwt/PR8 or AAmut/PR8 or treated with tunicamycin at $5 \mathrm{ug} / \mathrm{ml}(\mathrm{n}=3)$. Cells were collected at 16 and $24 \mathrm{~h}$ post infection RT-qPCR for UPR-induced genes DNAJB9 and DDIT3.

(d) A549 (upper panel) or LA4 (lower panel) were infected at a MOI of 0.01 with either AAwt/PR8 or AAmut/PR8. Supernatants were collected at indicated time post infection and viral titer determined by plaque assay.

(e) Primary human nasal epithelial cells (Mucilair) were infected at a MOI of 5 with AAwt/PR8 or AAmut/PR8 $(n=6)$ or treated with tunicamycin $(n=2)$ at $5 \mathrm{ug} / \mathrm{ml}$ for $8 \mathrm{~h}$. Cells were lysed at $24 \mathrm{~h}$ post infection and RT-qPCR for each respective gene was performed.

The statistical significances between AAwt/PR8 group versus AAmut/PR8 group were determined using one-way ANOVA with post-hoc Tukey's multiple comparison test for panels b-d and g and two-way ANOVA with the Geisser-Greenhouse correction and post-hoc Dunn's multiple comparisons test for panels e and f. ${ }^{*} p<0.05,{ }^{* *} p<0.01, * * * p<0.001,{ }^{*} p<0.05$ between mock and AAmut/PR8 groups; ns = non-significant. Graphs are representative of 23 independent experiments and indicate mean \pm SD. 